(1)

CrossMark

\title{
Incidence of non-pulmonary cancer and lung cancer by amount of emphysema and airway wall thickness: a community-based cohort
}

\author{
Ane Aamli Gagnat ${ }^{1}$, Miriam Gjerdevik ${ }^{2,3}$, Frode Gallefoss ${ }^{1}$, Harvey O. Coxson ${ }^{4}$, \\ Amund Gulsvik ${ }^{1}$ and Per Bakke ${ }^{1}$
}

Affiliations: ${ }^{1}$ Dept of Clinical Science, University of Bergen, Bergen, Norway. ${ }^{2}$ Dept of Global Public Health and Primary Care, University of Bergen, Bergen, Norway. ${ }^{3}$ Dept of Thoracic Medicine, Norwegian Registry for Chronic Obstructive Pulmonary Disease and Norwegian Registry for Long-Term Mechanical Ventilation, Haukeland University Hospital, Bergen, Norway. ${ }^{4}$ Dept of Radiology and Centre for Heart Lung Innovation, University of British Columbia, Vancouver, BC, Canada.

Correspondence: Ane Aamli Gagnat, Dept of Clinical Science, Haukeland University Hospital, Postboks 7800 5020 Bergen, Norway. E-mail: ane.aamli.gagnat@gmail.com; ane.aamliđstudent.uib.no

@ERSpublications

Emphysema is an independent risk factor for both non-pulmonary cancer and lung cancer http://ow.ly/SPR1308EgYn

Cite this article as: Aamli Gagnat A, Gjerdevik M, Gallefoss F, et al. Incidence of non-pulmonary cancer and lung cancer by amount of emphysema and airway wall thickness: a community-based cohort. Eur Respir J 2017; 49: 1601162 [https://doi.org/10.1183/13993003.01162-2016].

ABSTRACT There is limited knowledge about the prognostic value of quantitative computed tomography (CT) measures of emphysema and airway wall thickness in cancer.

The aim of this study was to investigate if using CT to quantitatively assess the amount of emphysema and airway wall thickness independently predicts the subsequent incidence of non-pulmonary cancer and lung cancer.

In the GenKOLS study of 2003-2005, 947 ever-smokers performed spirometry and underwent CT examination. The main predictors were the amount of emphysema measured by the percentage of low attenuation areas (\%LAA) on CT and standardised measures of airway wall thickness (AWT-PI10). Cancer data from 2003-2013 were obtained from the Norwegian Cancer Register. The hazard ratio associated with emphysema and airway wall thickness was assessed using Cox proportional hazards regression for cancer diagnoses.

During 10 years of follow-up, non-pulmonary cancer was diagnosed in $11 \%$ of the subjects with LAA $<3 \%$, in $19 \%$ of subjects with LAA $3-10 \%$, and in $17 \%$ of subjects with LAA $\geqslant 10 \%$. Corresponding numbers for lung cancer were $2 \%, 3 \%$ and $11 \%$, respectively. After adjustment, the baseline amount of emphysema remained a significant predictor of the incidence of non-pulmonary cancer and lung cancer. Airway wall thickness did not predict cancer independently.

This study offers a strong argument that emphysema is an independent risk factor for both nonpulmonary cancer and lung cancer.

Received: June 132016 | Accepted after revision: Jan 282017

Support statement: The GenKOLS study was funded by GlaxoSmithKline Research and Development Limited, UK. Funding information for this article has been deposited with the Open Funder Registry.

Conflict of interest: Disclosures can be found alongside this article at erj.ersjournals.com 


\section{Introduction}

Emphysema in patients with chronic obstructive pulmonary disease (COPD) is the strongest known imaging biomarker for lung cancer [1]. COPD is considered a systemic disease, in which $16 \%$ of patients show manifestations of systemic inflammation [2]. Given that inflammation in COPD is a potential mechanism for increased lung cancer risk in these patients, one might hypothesise that systemic inflammation in at least some COPD patients implies a higher risk of non-pulmonary cancer. To our knowledge, this hypothesis has not previously been tested [1].

Previous computed tomography (CT) studies have shown a positive correlation between emphysema and lung cancer by qualitative assessment of emphysema [3-7], whereas conflicting results exist concerning emphysema quantified automatically by software [7-9].

Lung CT examinations of patients with COPD also yield information on their airways. Limited data are available regarding whether airway wall thickness, as a marker of airway inflammation, is associated with an increased incidence of lung cancer as well as non-pulmonary malignancies.

We had access to a community sample of subjects with and without COPD with CT scanning of their lungs. These data were merged with data from the Norwegian Cancer Register from 2013 [10] to explore whether the CT-assessed amount of emphysema and airway wall thickness can predict subsequent incidence of both non-pulmonary cancer and lung cancer.

\section{Methods}

\section{Study subjects}

Subjects were aged $40-85$ years and had a smoking history of $\geqslant 2.5$ pack-years at baseline. The 947 subjects included in the current study had all participated in the GenKOLS (Genetic COPD) study conducted from January 2003 to January 2005 [11]. They represented approximately half of the GenKOLS sample $(n=1909)$ who received an optional high-resolution CT scan. Of these 947 subjects, 57 had been diagnosed with cancer prior to inclusion and four subjects left the country during follow-up. They were therefore omitted from further analyses. Thus, 886 subjects were eligible for the current analyses: 422 COPD cases and 464 controls. The GenKOLS study recruited subjects from two general population studies [12, 13] and a hospital patient register. Details on the study population are described elsewhere [14]. COPD was diagnosed when the post-bronchodilator forced expiratory volume in $1 \mathrm{~s}$ (FEV1) to forced vital capacity (FVC) ratio was $<0.70$ and $\mathrm{FEV}_{1}$ was $<80 \%$ predicted. Those without $\mathrm{COPD}$ required a post-bronchodilator FEV $1 / F V C \geqslant 0.70$ and $\mathrm{FEV}_{1} \geqslant 80 \%$ predicted. The examination at baseline in 2003-2005 included thorax $\mathrm{CT}$, pulmonary function tests and questionnaires on smoking habits.

\section{Quantitative interpreted computed tomography}

CT scans were performed with a GE LightSpeed Ultra CT scanner $(120 \mathrm{kVp}, 200 \mathrm{~mA}$; GE Healthcare, Milwaukee, WI, USA) at full inspiration using $1-\mathrm{mm}$ slice thickness at $20-\mathrm{mm}$ intervals. The extent of emphysema was assessed using the percentage of lung voxels with X-ray attenuation values $<-950$ Hounsfield units (percentage of low-attention areas (\%LAA)). This cut-off has been shown to be accurate for this CT acquisition technique [15]. The percentage of emphysema for the whole lung was calculated. Airway wall thickness is presented as the square root of the wall area for a standardised airway with an internal perimeter of $10 \mathrm{~mm}$. Details on the CT scanning are presented elsewhere [11].

\section{The Norwegian Cancer Register}

Every person in Norway diagnosed with cancer of any type is registered in the Norwegian Cancer Register. Registration is obligatory by law, both by clinical doctors and pathologists, thus securing completeness of the registry [10]. The inclusion rate is close to $100 \%$. Thus, subjects who developed cancer during the follow-up in our study were identified from the Norwegian Cancer Register. Lung cancers were defined by International Classification of Diseases 10th Revision (ICD-10) code C34 and non-pulmonary cancer was defined as all cancers outside the lung. The patients' identification numbers were given with permission from the Norwegian Data Inspectorate, the Norwegian Directorate of Health and Social Services and the Regional Ethical Committee for Medical Research. Data obtained included time of diagnosis, cancer histology and stage, and location of cancer at the time of diagnosis.

\section{Other variables}

Smoking variables included current smoking status, pack years and age at onset of smoking, all self-reported at baseline. Spirometry was performed according to the American Thoracic Society standards [16]. Reference values for FEV1 and FVC were local [17]. COPD patients included were categorised as stage 2-4 according to the Global Initiative for Chronic Obstructive Lung Disease (GOLD) 2007 classification, as defined by the percentage of predicted FEV1. Further, the new GOLD 2011 guidelines 
have an A-D grouping and are based on self-reported dyspnoea and symptoms (Modified British Medical Research Council (mMRC) Dyspnea Scale, COPD Assessment Test (CAT) score) and number of exacerbations in the last 12 months in addition to the percentage of predicted FEV1 [18].

\section{Statistical analysis}

Emphysema measured as \%LAA was the main predictor of interest and was categorised into three categories: low (LAA $<3 \%$ ), medium (LAA 3-10\%) and high (LAA $\geqslant 10 \%$ ). With the lack of suggested classifications of \%LAA stages, these categories were based on inspection of the quantile plot, as described in a previous study on the same population examining emphysema and mortality [19]. The amount of emphysema was also assessed with \%LAA as a continuous independent variable.

The hazard ratios associated with emphysema and airway wall thickness were estimated using Cox proportional hazards regression for cancer diagnoses. Kaplan-Meier plots were constructed. In the multivariate analyses we adjusted for sex, age, smoking status, age of onset of smoking, pack years and percentage of predicted FEV1. An overall comparison of the distribution of non-pulmonary cancers across the amount of emphysema was performed using Fisher's exact test. The a priori decided interactions between both emphysema and airway wall thickness and the variables sex, age, smoking status, pack years and COPD status, respectively, were tested by employing the likelihood ratio test. The proportionality assumption was checked using the post estimation command estat phtest in STATA.

All analyses were performed with STATA 13 (Stata Statistical Software: Release 13; StataCorp, College Station, TX, USA), and the two-sided significance level was conventionally set to 0.05 for all analyses.

\section{Results}

The subjects comprised $41 \%$ women, the mean \pm SD age was $59.4 \pm 10.3$ years and $48 \%$ had COPD at baseline (table 1). By inclusion criteria, all were ever-smokers. During the 10-year follow-up, 13\% were diagnosed with non-pulmonary cancer and $4 \%$ with lung cancer, while $0.9 \%$ received both diagnoses. Altogether, $88 \%$ of those diagnosed with lung cancer had COPD at baseline. Among the COPD patients, about half of those receiving a lung cancer diagnosis had GOLD stage D at baseline. Only 18\% of those not diagnosed with cancer had a LAA $\geqslant 10 \%$, whereas the corresponding numbers for those diagnosed with non-pulmonary cancer and lung cancer were $25 \%$ and $59 \%$, respectively.

Kaplan-Meier plots show the incidence estimates for non-pulmonary cancers and lung cancer by amount of emphysema (figure 1). Data show that the higher the amount of emphysema, the higher the risk of

\begin{tabular}{lccc}
\hline TABLE 1 Baseline characteristics by cancer status after follow-up & \\
Characteristics & Pulmonary cancer & Non-pulmonary cancer ${ }^{\#}$ & Non-cancers \\
\hline Subjects n & 34 & 119 & 741 \\
Male & 61.8 & 63.9 & 57.8 \\
Age years & $65.1 \pm 6.6$ & $64.4 \pm 9.3$ & $58.4 \pm 10.3$ \\
Current smokers & 44.1 & 30.3 & 47.8 \\
Age of onset of smoking years & $17.9 \pm 4.6$ & $18.5 \pm 4.5$ & $18.4 \pm 5.0$ \\
Pack-years, median (IQR) & $33.8(22-50)$ & $22.1(14-38)$ & $21.0(12-32)$ \\
Subjects without COPD & 11.8 & 47.9 & 54.5 \\
GOLD stages in COPD cases & & & \\
Stage A & 20.0 & 21.7 & 25.9 \\
Stage B & 26.7 & 45.0 & 29.9 \\
Stage C & 3.3 & 5.0 & 7.3 \\
Stage D & 50.0 & 28.3 & 36.9 \\
FEV ${ }^{\text {T }}$ & $54.3 \pm 21.4$ & $75.8 \pm 23.5$ & $75.7 \pm 24.8$ \\
Emphysema \%LAA & $15.7 \pm 13.2$ & $6.9 \pm 9.0$ & $5.5 \pm 9.5$ \\
Emphysema categories & & & \\
LAA <3\% & 26.5 & 51.3 & 66.5 \\
LAA 3-10\% & 14.7 & 23.5 & 15.9 \\
LAA $\geqslant 10 \%$ & 58.8 & 25.2 & 17.5 \\
AWT-Pi10 mm & $4.83 \pm 0.35$ & $4.85 \pm 0.32$ & $4.85 \pm 0.33$
\end{tabular}

Data are presented as $\%$ or mean $\pm S D$, unless otherwise indicated. IQR: interquartile range; COPD: chronic obstructive pulmonary disease; GOLD: Global Initiative for Chronic Obstructive Lung Disease; FEV1: forced expiratory volume in $1 \mathrm{~s}$; LAA: low-attenuation area; AWT-Pi10: airway wall thickness (square root of wall area for airway with internal perimeter of $10 \mathrm{~mm}$ ). ${ }^{*}$ : 8 persons had both lung cancer and non-pulmonary cancer; १: percentage of predicted post-bronchodilator FEV1. 

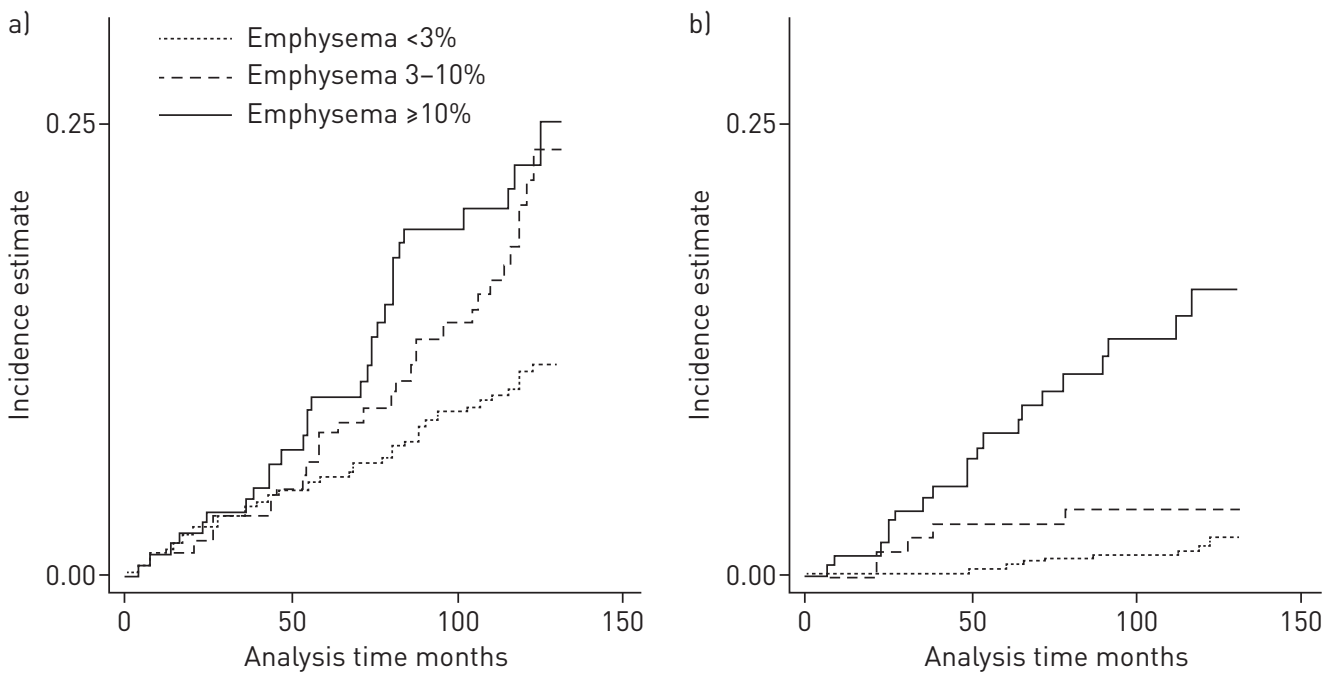

FIGURE 1 Kaplan-Meier curve for the incidence of al non-pulmonary and b) lung cancer by amount of emphysema. The figure shows censored data.

non-pulmonary cancer and lung cancer. This is consistent with crude analyses, not accounting for censoring. Without taking censoring into account, $11 \%$ of the subjects in the lowest \%LAA emphysema category got non-pulmonary cancer, compared with $19 \%$ in the middle emphysema category and $17 \%$ in the highest category. The corresponding numbers for those who received a lung cancer diagnosis were $2 \%$, $3 \%$ and $11 \%$, respectively.

Table 2 shows the distribution of the various non-pulmonary cancers. Although the incidence of the non-pulmonary cancers increased with increasing amount of emphysema, the distribution of non-pulmonary cancer did not differ between the amounts of emphysema (Fisher's exact test $\mathrm{p}=0.26$ ). Airway wall thickness at baseline was not related to either lung cancer or non-pulmonary cancer (tables 3 and 4).

The univariate hazard ratios showed that older age, pack years, COPD status and amount of emphysema at baseline were significantly related to both incidences of non-pulmonary cancer and lung cancer (table 3). The hazard ratio for lung cancer by percentage of predicted FEV1 also reached a level of significance; the lower the percentage of predicted FEV1, the higher the hazard ratio (table 3 ).

After adjusting for sex, age and smoking (i.e. ex-versus current smoking, pack years and age of onset of smoking), the baseline amount of emphysema was still a significant predictor of incidence of both non-pulmonary cancer and lung cancer (table 4).

\section{TABLE 2 Distribution of non-pulmonary cancers by level of emphysema}

\begin{tabular}{lcccc} 
Cancer type & ICD10 & LAA $<\mathbf{3 \%}$ & LAA 3-10\% & LAA $\geqslant \mathbf{1 0 \%}$ \\
\hline Subjects n & & 61 & 28 & 30 \\
1) Lip, oral cavity, pharynx & C00-C14 & 1.6 & 3.6 & 3.3 \\
2) Digestive organs & C15-C26 & 13.1 & 42.9 & 20.0 \\
3) Respiratory system, not lung & C32+C45 & 3.3 & 0.0 & 0.0 \\
4) Skin & C43-C44 & 8.2 & 3.6 & 16.7 \\
5) Retroperitoneum, peritoneum & C48 & 1.6 & 0.0 & 0.0 \\
6) Breast cancer & C50 & 16.4 & 7.1 & 6.7 \\
7) Female genital organs & C51-C58 & 4.9 & 7.1 & 0.0 \\
8) Male genital organs & C60-63 & 24.6 & 10.7 & 13.3 \\
9) Urinary tract & C64-C68 & 8.2 & 14.3 & 20.0 \\
10) Eye, brain, central nervous system & C69-C72 & 4.9 & 0.0 & 6.7 \\
11) Thyroid, other endocrine gland & C73-C75 & 3.3 & 0.0 & 0.0 \\
12) Unknown primary site & C76+C83 & 1.6 & 0.0 & 3.3 \\
13) Bone marrow & C90-92+D46-47 & 8.2 & 10.7 & 10.0 \\
Sum & & 100 & 100 & 100
\end{tabular}

Data are presented as \%, unless otherwise indicated. LAA: low-attenuation area; ICD10: International Classification of Diseases 10th Revision. 
TABLE 3 Unadjusted analyses of predictors for incidence of pulmonary and non-pulmonary cancer from baseline to December 2013

\begin{tabular}{lcc} 
Characteristics & Pulmonary cancer & Non-pulmonary cancer \\
\hline Male & $1.18(0.59-2.36)$ & $1.3(0.90-1.89)$ \\
Age & $1.07^{* * *}(1.04-1.11)$ & $1.07^{* * *}(1.05-1.10)$ \\
Current smoking & $0.87(0.44-1.72)$ & $0.48^{* * *}(0.32-0.71)$ \\
Age of onset of smoking & $0.98(0.90-1.06)$ & $1.01(0.97-1.04)$ \\
Pack years & $1.03^{* * *}(1.02-1.05)$ & $1.01(1.00-1.02)$ \\
COPD cases versus controls & $9.83^{* * *}(3.46-27.93)$ & $1.45^{*}(1.01-2.08)$ \\
FEV ${ }^{\#}$ & $0.96^{* * *}(0.95-0.98)$ & $1(0.99-1.00)$ \\
Emphysema categories & & Ref. \\
LAA $<\%$ & Ref. & $1.92^{* *}(1.22-3.01)$ \\
LAA 3-10\% & $2.32(0.78-6.93)$ & $2.18^{* * *}(1.41-3.39)$ \\
LAA $\geqslant 10 \%$ & $9.56^{* * *}(4.34-21.05)$ & $1.04(0.59-1.82)$ \\
AWT-Pi10 & $0.82(0.29-2.34)$ & $1.24^{*}(1.05-1.46)$ \\
Emphysema per 10\% increase & $1.95^{* * *}(1.58-2.41)$ & \\
\hline
\end{tabular}

Data are presented as hazard ratio (95\% CI). COPD: chronic obstructive pulmonary disease; FEV1: forced expiratory volume in $1 \mathrm{~s}$; LAA: low-attenuation area; AWT-Pi10: airway wall thickness Isquare root of wall area for airway with internal perimeter of $10 \mathrm{~mm}$ ). "\#: percentage of predicted post-bronchodilator FEV1. ${ }^{*}: p<0.05 ;^{* *}: p<0.01 ;{ }^{* * *}: p<0.001$.

Finally, we added the a priori determined interaction terms to the equation. None of them reached the level of significance. We also repeated the analyses removing those patients with severe GOLD stages (stages $\mathrm{C}$ and $\mathrm{D}$ ). The coefficients remained unchanged, indicating that the amount of emphysema was also related to an increased risk of lung cancer in the healthier part of the population (those with non-COPD and COPD stage $\mathrm{A}$ and $\mathrm{B}$, results not shown).

We found no relationship between the location of emphysema in the lung and the location of the cancer. Nor did we find any relationship between emphysema and stage/metastasis in lung cancers, or between emphysema and the histological subtype of lung cancer (results not shown).

\section{Discussion}

The main findings of this prospective community-based study in subjects with and without COPD were as follows: 1) an increased amount of emphysema predicted an increased risk of non-pulmonary cancer, as well as lung cancer; and 2) airway wall thickness was related to neither lung cancer nor non-pulmonary cancer.

To our knowledge, this is the first study to show that emphysema predicts cancer outside the lungs. We observed that emphysema was a predictor of non-pulmonary cancer after adjusting for sex, age and smoking.

Several mechanisms may explain the association between emphysema and non-pulmonary cancer. First, COPD, with emphysema as a phenotype, is regarded as a systemic disease with one in six having chronic systemic inflammation [2]. Many inflammatory signalling pathways are activated in several types of cancer, linking chronic inflammation to tumour genesis [20]. Many inflammatory mediators, including cytokines,

TABLE 4 Associations of emphysema (and airway wall thickness) with incidence of pulmonary and non-pulmonary cancer from baseline to December 2013

\begin{tabular}{lcc} 
& Pulmonary cancer & Non-pulmonary cancer \\
\hline Emphysema per $\mathbf{1 0 \%}$ increase & $1.41 *(1.00-1.97)$ & $1.13(0.89-1.44)$ \\
Emphysema categories & Ref. & Ref. \\
LAA $<3 \%$ & $1.21(0.37-9.98)$ & $1.55(0.93-2.57)$ \\
LAA $3-10 \%$ & $3.33 *(1.04-10.61)$ & $2.10 *(1.14-3.87)$ \\
LAA $\geqslant 10 \%$ & $0.39(0.12-1.29)$ & $0.82(0.42-1.59)$ \\
AWT-Pi10 &
\end{tabular}

Data are presented as hazard ratio $(95 \% \mathrm{Cl})$. Adjusted for sex, age, percentage of predicted forced expiratory volume in $1 \mathrm{~s}$, pack years, smoking status and age of onset of smoking. LAA: low-attenuation area; AWT-Pi10: airway wall thickness (square root of wall area for airway with internal perimeter of $10 \mathrm{~mm}) . *$ : $p<0.05$. 
chemokines, free radicals, growth factors and enzymes like matrix metalloproteinases may act to create a favourable microenvironment for the development of tumours [20]. Several of the signalling molecules mentioned above might be elevated in patients with COPD $[2,21]$. Second, common genetic and epigenetic factors might also be responsible for both emphysema and non-pulmonary cancer [1]. Examples of such genes are those that code for telomerase reverse transcriptase (TERT) and NF- $\mathrm{B}$ [22, 23]. Third, emphysema has been considered a marker of accelerated ageing in COPD [24]. Hence, emphysema might be an indicator of senescence of the lungs in the same way that muscle wasting and osteoporosis in COPD might be indicators of senescence in other organ systems. Accelerated ageing is related to increased risk of cancer development [25]. Consequently, both emphysema and increased risk of cancer could be part of an accelerated ageing process in patients with COPD. Finally, there could be other environmental risk factors for both emphysema and malignancies. An example is asbestos exposure, which can cause emphysema [26] and both pulmonary and non-pulmonary malignancies, at least in heavily exposed workers [27].

We also observed a relationship between quantitatively assessed emphysema and the risk of lung cancer. This is in line with previous studies using qualitative CT assessments [2-6], whereas studies using quantitative scoring have provided contradictory findings [7-9, 28]. Why did we find a positive emphysema-lung cancer correlation while other quantitative studies have failed to do so? The other quantitative studies were nested case-control studies sampled from lung cancer screening trials, whereas the present study was a community-based cohort of ever-smokers followed for 10 years. It might be that lung cancer screening trials do not represent the population with emphysema in general [3]. Even though the authors using qualitative assessments often state that the CT readers were unaware of the lung cancer diagnosis, it is possible that other radiographic characteristics, such as nodules, might have biased the readers' assessments of emphysema [3]. Our study using quantitative emphysema assessment is a strong argument for a true increased risk of lung cancer in those with emphysema. We observed a dose-response relationship between emphysema and lung cancer, which further strengthens this argument.

Several mechanisms may explain the association between emphysema and lung cancer, most of them being the same as stated for the association between emphysema and non-pulmonary cancer. These mechanisms include smoking as a prevalent risk factor for both emphysema and lung cancer through chronic inflammation and enzymatic imbalance. Emphysema may also cause scarring and repair processes that can lead to lung cancer. Finally, impaired mucociliary clearance in emphysema may cause an accumulation of carcinogens in the lungs.

We did not observe any relationship between airway wall thickness in terms of Pi10 and incidence of lung cancer. This is in line with the findings of a nested case-control study that included 117 matched pairs of lung cancer cases and controls sampled from a screening trial [7]. Other reports from our study population have shown that increased Pi10 is an independent predictor of respiratory symptoms, and also of respiratory mortality in those with severe emphysema [19, 29]. Airway wall thickening in COPD corresponds with chronic inflammation, but also with structural modifications including hypertrophy and hyperplasia of parietal glands, enlargement of goblet cells and mucus production [30, 31]. It may be hard to determine if airway wall thickness as measured by Pi10 reflects active, stable or burned out disease [32]. Hence, the lack of an association between Pi10 and lung cancer may indicate that Pi10 is an unspecific measure of airway inflammation.

The present study has several important strengths. First, it is a large single-centre study, which allows for extensive adjustment for important confounders. The participants were not sampled from a cancer screening trial, but from a community-based sample followed for more than 8000 person years. Second, all the CT scans were performed using the same scanner and were quantitatively interpreted. Third, this is a prospective study in which all the lung cancers and non-pulmonary cancers were incident cases, diagnosed after the CT scanning. Fourth, the cancer diagnoses were taken from the Norwegian Cancer Register with a close to $100 \%$ inclusion rate and histologically verified cancer diagnosis [10]. Finally, we also adjusted for the percentage of predicted FEV1 without this affecting the overall findings of the study. However, it could be argued that FEV1 is not a potential confounder in the emphysema-cancer relationship because FEV1 does not cause emphysema but rather is at least partly an effect of emphysema.

Some limitations should also be acknowledged. First, the study was primarily designed to examine COPD patients. Thus, the number of incident cancer cases was small. Further, the design did not allow for a correlation assessment of the amount of emphysema versus the type of lung cancer. Second, it could be argued that COPD is mainly a distal airway disease whereas the Pi10 measurements reflect central airway conditions. However, findings in the central airways correspond to those in the distal airways [33]. Third, the CT scans were acquired using a high-resolution CT technique, which was common during the time of the data acquisition for this study. However, all the CT scans were performed using a standard protocol and the measurements of emphysema have been shown to be reproducible using a standard protocol [34]. 
Moreover, other studies have shown that limited CT scans can produce values of overall emphysema that are comparable to complete volumetric CT scans [35]. Fourth, the study did not include never-smokers, which prevents us from generalising the results to never-smokers. However, smokers with as low a smoking consumption as 2.5 pack years were included. Fifth, the CT assessment did not include the type of emphysema. Sixth, the study did not include GOLD stage I, which in some studies has shown to have the highest incidence of lung cancer [36].

It is well known that COPD patients have an increased risk of lung cancer. This study indicates that emphysema should be taken into account when evaluating those at high risk for not only lung cancer, but also non-pulmonary cancer. Further studies should be conducted to see if these patients could benefit from screening programmes.

In conclusion, this study offers a strong argument that emphysema is an independent risk factor for non-pulmonary cancer and lung cancer. Airway wall thickness in terms of Pi10 did not predict either non-pulmonary cancer or lung cancer.

\section{Acknowledgements}

Author contributions: A. Aamli Gagnat performed the statistical analyses, and drafted and revised the paper. M. Gjerdevik performed the statistical analyses, and drafted and revised the paper. F. Gallefoss contributed significantly in the discussion, and drafted and revised the paper. H.O. Coxson took part in the data collection, analysed the CT scans, and revised and drafted the paper. A. Gulsvik designed the study, took part in the data collection, and drafted and revised the paper. P. Bakke designed the study, took part in the data collection, and drafted and revised the paper.

\section{References}

1 Zulueta JJ. Emphysema and lung cancer. More than a coincidence. Ann Am Thorac Soc 2015; 12: 1120-1121.

2 Agustí A, Edwards LD, Rennard SI, et al. Persistent systemic inflammation is associated with poor clinical outcomes in COPD: a novel phenotype. PLoS One 2012; 7: e37483.

3 Wilson DO, Weissfeld JL, Balkan A, et al. Association of radiographic emphysema and airflow obstruction with lung cancer. Am J Respir Crit Care Med 2008; 178: 738-744.

4 de Torres JP, Bastarrika G, Wisnivesky JP, et al. Assessing the relationship between lung cancer risk and emphysema detected on low-dose CT of the chest. Chest 2007; 132: 1932-1938.

5 Zulueta JJ, Wisnivesky JP, Henschke CI, et al. Emphysema scores predict death from COPD and lung cancer. Chest 2012; 141: 1216-1223.

6 Li Y, Swensen SJ, Karabekmez LG, et al. Effect of emphysema on lung cancer risk in smokers: a computed tomography-based assessment. Cancer Prev Res (Phila) 2011; 4: 43-50.

7 Wilson DO, Leader JK, Fuhrman CR, et al. Quantitative computed tomography analysis, airflow obstruction, and lung cancer in the Pittsburgh Lung Screening Study. J Thorac Oncol 2011; 6: 1200-1205.

8 Maldonado F, Bartholmai BJ, Swensen SJ, et al. Are airflow obstruction and radiographic evidence of emphysema risk factors for lung cancer? A nested case-control study using quantitative emphysema analysis. Chest 2010; 138: 1295-1302.

9 Sverzellati N, Cademartiri F, Bravi F, et al. Relationship and prognostic value of modified coronary artery calcium score, FEV1, and emphysema in lung cancer screening population: the MILD trial. Radiology 2012; 262: 460-467.

10 Larsen IK, Småstuen M, Johannesen TB, et al. Data quality at the Cancer Registry of Norway: an overview of comparability, completeness, validity and timeliness. Eur J Cancer 2009; 45: 1218-1231.

11 Grydeland TB, Dirksen A, Coxson HO, et al. Quantitative computed tomography: emphysema and airway wall thickness by sex, age and smoking. Eur Respir J 2009; 34: 858-865.

12 Eagan TM, Gulsvik A, Eide GE, et al. Remission of respiratory symptoms by smoking and occupational exposure in a cohort study. Eur Respir J 2004; 23: 589-594.

13 Brogger J, Eagan T, Eide GE, et al. Bias in retrospective studies of trends in asthma incidence. Eur Respir J 2004; 23: 281-286.

14 Sørheim IC, Johannessen A, Grydeland TB, et al. Case-control studies on risk factors for chronic obstructive pulmonary disease: how does the sampling of the cases and controls affect the results? Clin Respir J 2010; 4: 89-96.

15 Gevenois PA, de Maertelaer V, De Vuyst P, et al. Comparison of computed density and macroscopic morphometry in pulmonary emphysema. Am J Respir Crit Care Med 1995; 152: 653-657.

16 Standards for the diagnosis and care of patients with chronic obstructive pulmonary disease. American Thoracic Society. Am J Respir Crit Care Med 1995; 152: S77-S121.

17 Gulsvik A, Tosteson T, Bakke P, et al. Expiratory and inspiratory forced vital capacity and one-second forced volume in asymptomatic never-smokers in Norway. Clin Physiol 2001; 21: 648-660.

18 Johannessen A, Nilsen RM, Storebø M, et al. Comparison of 2011 and 2007 Global Initiative for Chronic Obstructive Lung Disease guidelines for predicting mortality and hospitalization. Am J Respir Crit Care Med 2013; 188: 51-59.

19 Johannessen A, Skorge TD, Bottai M, et al. Mortality by level of emphysema and airway wall thickness. Am J Respir Crit Care Med 2013; 187: 602-608.

20 Fernandes JV, Cobucci RN, Jatoba CA, et al. The role of the mediators of inflammation in cancer development. Pathol Oncol Res 2015; 21: 527-534.

21 Faner R, Tal-Singer R, Riley JH, et al. Lessons from ECLIPSE: a review of COPD biomarkers. Thorax 2014; 69: $666-672$.

22 Akincilar SC, Unal B, Tergaonkar V. Reactivation of telomerase in cancer. Cell Mol Life Sci 2016; 73: 1659-1670.

23 Calado RT. Telomeres in lung diseases. Prog Mol Biol Transl Sci 2014; 125: 173-183.

24 Mercado N, Ito K, Barnes PJ. Accelerated ageing of the lung in COPD: new concepts. Thorax 2015; 70: 482-489.

25 Shay JW. Role of telomeres and telomerase in aging and cancer. Cancer Discov 2016; 6: 584-593. 
Vehmas T, Oksa P. Chest HRCT signs predict deaths in long-term follow-up among asbestos exposed workers. Eur J Radiol 2014; 83: 1983-1987.

27 Antwi SO, Eckert EC, Sabaque CV, et al. Exposure to environmental chemicals and heavy metals, and risk of pancreatic cancer. Cancer Causes Control 2015; 26: 1583-1591.

28 Oelsner EC, Carr JJ, Enright PL, et al. Per cent emphysema is associated with respiratory and lung cancer mortality in the general population: a cohort study. Thorax 2016; 71: 624-632.

29 Grydeland TB, Dirksen A, Coxson HO, et al. Quantitative computed tomography measures of emphysema and airway wall thickness are related to respiratory symptoms. Am J Respir Crit Care Med 2010; 181: 353-359.

30 O'Shaughnessy TC, Ansari TW, Barnes NC, et al. Inflammation in bronchial biopsies of subjects with chronic bronchitis: inverse relationship of CD8+ T lymphocytes with FEV1. Am J Respir Crit Care Med 1997; 155: 852-857.

31 Di Stefano A, Turato G, Maestrelli P, et al. Airflow limitation in chronic bronchitis is associated with T-lymphocyte and macrophage infiltration of the bronchial mucosa. Am J Respir Crit Care Med 1996; 153: 629-632.

32 Hogg JC, McDonough JE, Suzuki M. Small airway obstruction in COPD: new insights based on micro-CT imaging and MRI imaging. Chest 2013; 143: 1436-1443.

33 Nakano Y, Wong JC, de Jong PA, et al. The prediction of small airway dimensions using computed tomography. Am J Respir Crit Care Med 2005; 171: 142-146.

34 Yuan R, Mayo JR, Hogg JC, et al. The effects of radiation dose and CT manufacturer on measurements of lung densitometry. Chest 2007; 132: 617-623

35 Hoffman EA, Jiang R, Baumhauer H, et al. Reproducibility and validity of lung density measures from cardiac CT Scans--The Multi-Ethnic Study of Atherosclerosis (MESA) Lung Study. Acad Radiol 2009; 16: 689-699.

36 Sin DD, Anthonisen NR, Soriano JB, et al. Mortality in COPD: role of comorbidities. Eur Respir J 2006; 28: 1245-1257. 